\title{
Intensity-modulated radiation therapy for elderly patients (aged $\geq 75$ years) with localized prostate cancer: Comparison with younger patients (aged $<75$ years)
}

\author{
HIDEKAZU TANAKA ${ }^{1}$, YUKA NAKASHIMA ${ }^{1}$, MASAYA ITO ${ }^{1}$, TAKAHIRO YAMAGUCHI ${ }^{1}$, \\ KAE ESAKI $^{1}$, SHINGO KAMEI ${ }^{2}$, SATOSHI ISHIHARA ${ }^{2}$, MASAHIDE HAYASHI ${ }^{3}$, \\ SHINICHI OGAWA ${ }^{3}$, SATOSHI GOSHIMA ${ }^{1}$ and MASAYUKI MATSUO ${ }^{1}$ \\ ${ }^{1}$ Department of Radiology, Gifu University Hospital, Gifu 501-1194; \\ Departments of ${ }^{2}$ Urology and ${ }^{3}$ Radiology, Kizawa Memorial Hospital, Minokamo, Gifu 505-8503, Japan
}

Received November 5, 2018; Accepted February 12, 2019

DOI: $10.3892 /$ mco.2019.1810

\begin{abstract}
The aim of the present study was to evaluate the efficacy and safety of intensity-modulated radiation therapy (IMRT) for elderly patients with prostate cancer (age $\geq 75$ years) compared with younger patients ( $<75$ years). The numbers of patients enrolled into the elderly and younger groups were 238 and 853, respectively. More than half of the patients in the elderly group were high-risk, and the total risk of the elderly group was higher than that of younger group. The median follow-up periods for the elderly and younger groups were 42 (range, 2-108) and 49 (range, 2-120) months, respectively. All patients were treated with IMRT at a dose of 74-78 Gy with or without androgen-deprivation therapy. The biochemical failure-free rates (BFFRs) at 3-year follow-up for the elderly and younger groups were 93.3 and $95.7 \%$, respectively; there was no significant difference between the 2 groups in regard to the BFFR. The clinical failure-free rates (CFFR) at 3-year follow-up for the elderly and younger groups was 95.8 and $98.5 \%$, respectively; the 2 groups did not differ significantly in regard to the CFFR. The cumulative incidence rates of gastrointestinal toxicity (grade $\geq 2$ ) and genitourinary toxicity (grade $\geq 2$ ) at 3 -year follow-up were 10.5 and $1.3 \%$, respectively; there was no significant difference between the elderly and younger groups. It was concluded that in prostate cancer patients aged 75 years or older, IMRT has a treatment effect equivalent to that in patients $<75$ years old; adverse events are also comparable.
\end{abstract}

Correspondence to: Dr Hidekazu Tanaka, Department of Radiology, Gifu University Hospital, 1-1 Yanagido, Gifu 501-1194, Japan E-mail: htanaka-gif@umin.ac.jp

Key words: prostate cancer, external beam radiation therapy, intensity-modulated radiation therapy, elderly patients, adverse events

\section{Introduction}

An estimated 1,095,000 men worldwide were diagnosed with prostate cancer in 2012, resulting in 307,000 deaths (1). In Japan, the incidence of prostate cancer was estimated of 86,100 cases in 2017 (2), and in 2015, 11,326 patients died of prostate cancer; the proportion of death from prostate cancer was larger in elderly patients (age 70 and over) (3).

There are several options to treat prostate cancer, such as surgery, external beam radiation therapy (EBRT), brachytherapy, and androgen-deprivation therapy (ADT), excluding active surveillance and watchful waiting. Patients may receive one or a combination of these treatments (4). Among them, EBRT is less invasive than surgery or brachytherapy and has curability. Intensity-modulated radiation therapy (IMRT), a technique of EBRT, is especially effective and has lower rates of gastrointestinal (GI) adverse events than conventional EBRT (5-9). The safety of IMRT also allows dose escalation, leading to better tumor control (10). However, elderly patients were more likely to receive ADT alone (11). A recent study with elderly patients reported that the conservative management with ADT alone does not improve the overall survival rate (OS) (12).

According to data collected in 2016, the life expectancy of 75- and 80-year-old Japanese men is 12.14 and 8.92 years, respectively (13).

This study aimed to evaluate the efficacy and safety of IMRT for elderly prostate cancer patients (age more than or equal to 75 years) compared with younger patients (age less than 75 years).

\section{Materials and methods}

Patients. From March 2006 to July 2014, 1,252 prostate cancer patients were treated with IMRT at our hospital. Exclusion criteria included presence of lymph node metastases or distant metastases; furthermore, patients whose prostate-specific antigen (PSA) levels were not measured at least once after IMRT were excluded. Histologically, all tumors were found to be adenocarcinomas. Among the 1,091 patients, 238 patients 
Table I. Patient characteristics.

\begin{tabular}{|c|c|c|c|}
\hline Characteristics & Age $\geq 75$ years $(n=238)$ & Age $<75$ years $(n=853)$ & P-value \\
\hline Median age (range), years & $77(75-87)$ & $69(39-74)$ & - \\
\hline Median initial PSA (range), ng/ml & $9.59(4.09-356.00)$ & $8.27(2.65-370.00)$ & 0.1520 \\
\hline \multicolumn{4}{|l|}{ Initial PSA ng/ml, n (\%) } \\
\hline$<10$ & $125(52.5)$ & $505(59.2)$ & \\
\hline$\geq 10, \leq 20$ & 66 (27.7) & $214(25.1)$ & \\
\hline$>20$ & $47(19.7)$ & $133(15.6)$ & \\
\hline T Stage, n (\%) & & & 0.0017 \\
\hline $\mathrm{T} 1 \mathrm{c}$ & $76(31.9)$ & $351(41.1)$ & \\
\hline $\mathrm{T} 2 \mathrm{a}$ & $69(29.0)$ & $262(30.7)$ & \\
\hline $\mathrm{T} 2 \mathrm{~b}$ & $12(9.7)$ & $26(3.0)$ & \\
\hline $\mathrm{T} 2 \mathrm{c}$ & $33(13.9)$ & $92(10.8)$ & \\
\hline $\mathrm{T} 3 \mathrm{a}$ & $41(17.2)$ & $81(9.5)$ & \\
\hline $\mathrm{T} 3 \mathrm{~b}$ & $4(1.7)$ & $35(4.1)$ & \\
\hline $\mathrm{T} 4$ & $3(1.3)$ & $6(0.7)$ & \\
\hline Gleason's score, n (\%) & & & 0.0815 \\
\hline$\leq 6$ & $56(23.5)$ & $260(30.5)$ & \\
\hline 7 & $111(46.6)$ & $371(43.5)$ & \\
\hline 8 & $36(15.1)$ & $139(16.3)$ & \\
\hline 9 & $5(2.1)$ & $74(8.7)$ & \\
\hline 10 & $14(1.3)$ & $9(1.1)$ & \\
\hline D'Amico classification, n (\%) & & & 0.0055 \\
\hline Low risk & $31(13.0)$ & $174(20.4)$ & \\
\hline Intermediate risk & $78(32.8)$ & $338(39.6)$ & \\
\hline High risk & $129(54.5)$ & $341(40.0)$ & \\
\hline ADT use, n (\%) & & & $<0.0001$ \\
\hline Yes & $170(71.4)$ & $476(55.8)$ & \\
\hline No & 68 (28.6) & $377(44.2)$ & \\
\hline
\end{tabular}

PSA, prostate-specific antigen; ADT, androgen-deprivation therapy.

were aged 75 years or older, the younger group comprised 853 patients. Patient characteristics are listed in Table I. This study was approved by the Ethics Committee of Kizawa Memorial Hospital. All patients provided written informed consent before receiving radiotherapy.

According to the classification of D'Amico et al (14), patients were stratified into three risk groups. More than half of the patients in the elder group were high-risk, and the total risk of the elder group was higher than that of the younger group. The rate of ADT use administration was higher in the elder group than in the younger group. The median follow-up periods of the elder and younger groups were 42 (range, 2-108) and 49 (range, 2-120) months, respectively.

Treatment planning. One hour after urine collection, each patients was positioned supine with the immobilization devices and computed tomography (CT) scanning was performed. Axial CT scans of 2.5-mm thick sections were obtained from the superior border of the sacroiliac joint to $5 \mathrm{~cm}$ below the ischial tuberosity. TomoTherapy Treatment Planning System (TomoTherapy Inc., Madison, WI, USA) is a radiation delivery system that combines dynamic IMRT with an image-guided radiation therapy system. IMRT involving helical tomotherapy (HT) was planned using an inverse-planning approach. For T1-T3a cancer patients, the clinical target volume (CTV) was defined as the prostate and proximal portions of the seminal vesicles. For T3b cancer patients, the CTV included the prostate and entire seminal vesicles. The planning target volume (PTV) was set by expanding the CTV by $5 \mathrm{~mm}$ in all directions. Patients at low- and intermediate-risk with biopsy-positive core rate $\leq 50 \%$ were irradiated with 74 Gy in 37 fractions. Patients at intermediate-risk with biopsy-positive core rate $>50 \%$ were irradiated with 76 Gy in 38 fractions. For high-risk patients, the prescribed dose was 78 Gy in 39 fractions. Radiation therapy was administered five times a week. The dose limits for PTV were as follows: the volume of PTV receiving $95 \%$ of the prescribed dose (V95) was $>90 \%$ (preferably $>95 \%$ ); the volume of the PTV receiving at least $90 \%$ of the prescribed dose (V90) was $>96 \%$ (preferably $>98 \%$ ); and maximum dose to the PTV was $<110 \%$ of the prescribed dose. The rectum was delineated from $15 \mathrm{~mm}$ superior to $15 \mathrm{~mm}$ inferior to the PTV. Rectal wall thickness and bladder wall 


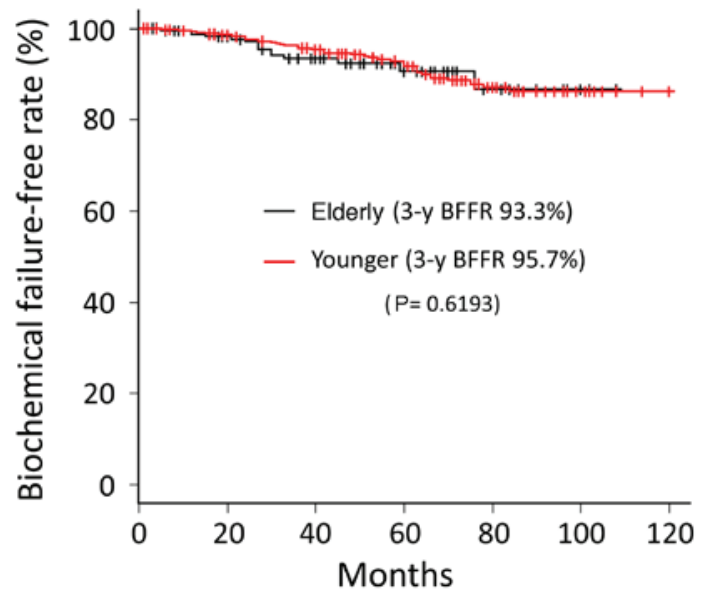

Figure 1. Biochemical failure-free rate for the elderly and younger groups. BFFR, biochemical failure-free rate; y, year.

thickness, both of $3 \mathrm{~mm}$, were created. The dose constrains for the rectum were $\mathrm{V} 40<60 \%, \mathrm{~V} 60<30 \%, \mathrm{~V} 70<20 \%$ and $\mathrm{V} 78<1 \%$. The dose constrains for the bladder were $\mathrm{V} 40<60 \%$ and $\mathrm{V} 70<30 \%$. Vx was defined as the percentage of structure volume receiving at least one dose of ' $\mathrm{x}$ ' Gy. Megavoltage CT image-guided verification was carried out every day prior to each treatment. A 6-MV photon beam was used for treatment.

ADT was started before IMRT and was continued during IMRT for patients in the intermediate-, and high-risk groups. Neoadjuvant ADT was initiated 3-6 months before IMRT. For high-risk patients, adjuvant ADT was continued for up to 2 years.

Evaluation. Biochemical failure was defined as PSA nadir plus $2 \mathrm{ng} / \mathrm{ml}$ according to the Phoenix criteria (15). In patients suspected of having local recurrence, magnetic resonance imaging (MRI) was performed and any MRI positive lesions were diagnosed using. A clinical failure was defined as the presence of local recurrence or metastases, and was confirmed by imaging, such as CT, MRI, or bone scintigraphy, in addition to checking PSA elevation. The examination was repeatedly conducted in patients with biochemical failure at appropriate timing, such as upon further elevation of PSA or appearance of symptom. Failure-free rates and survival rates were calculated from the completion date of IMRT to the occurrence of any event. We calculated the biochemical failure-free rate (BFFR), clinical failure-free rate (CFFR), and OS rates using the Kaplan-Meier method. Comparisons between the groups were conducted using the log-rank test. Using the Kaplan-Meier method, the cumulative incidence rate of grade $\geq 2$ GI and genitourinary (GU) toxicities were calculated according to the Common Terminology Criteria for Adverse Events (v4.0) (16). At our hospital, a radiation oncologist and urologist interviewed the patient regarding presence or absence of rectal bleeding upon each visit after IMRT. Moreover, a urologist performed digital rectal examination at every visit. When rectal bleeding is present, patients are required to undergo colonoscopy.

Comparison between clinical factors of the two groups was performed using the chi-square test and R 2.13.0 software (www.r-project.org/). $\mathrm{P}<0.05$ was considered to indicate a statistically significant difference.

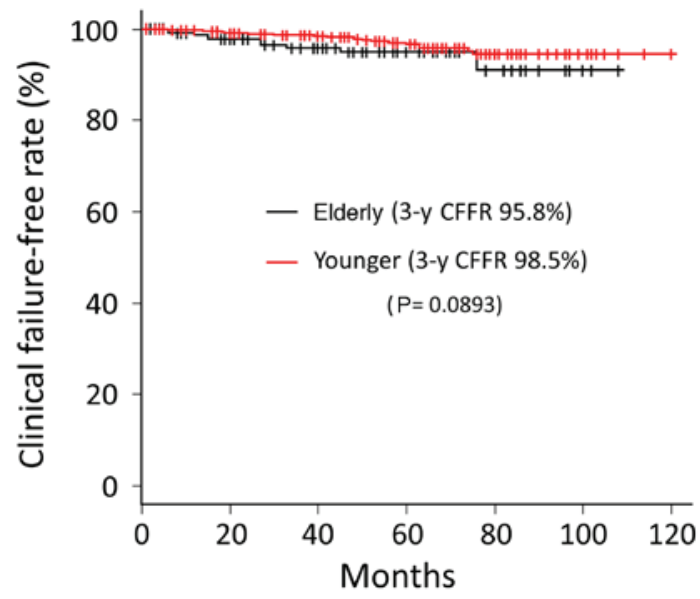

Figure 2. Clinical failure-free rate for the elderly and younger groups. CFFR, clinical failure-free rate; $y$, year.

\section{Results}

Biochemical failure was observed in 15 (6.3\%) patients in the elder group. The BFFRs at 3-year follow-up for elder and younger groups were 93.3 and $95.7 \%$, respectively. There was no significant difference between the two groups in BFFR $(\mathrm{P}=0.6193)$ (Fig. 1).

Ten $(4.2 \%)$ cases of clinical failure were observed in the elder group. The first failure sites were pelvic node, bone, and lungs in five, four, and one patient, respectively. Local failure was not observed in the elder group during the follow-up period. Conversely, the sites of first failure in the younger group were local (prostate), pelvic node, para-aortic node, and bone in eight, five, one, and nine patients, respectively. The CFFR at 3-year follow-up for elder and younger groups were 95.8 and $98.5 \%$, respectively. No significant difference was observed between the two groups $(\mathrm{P}=0.0893)$ (Fig. 2).

Five $(2.1 \%)$ patients in the elder group died during the follow-up period. Among them, two died due to prostate cancer, two died due to another cancer, and one died due to pneumonia. The OS rates at 3-year follow-up for elder and younger groups were 98.9 and $99.5 \%$, respectively; the difference between the two groups was statistically significant $(\mathrm{P}=0.0302)$ (Fig. 3). We compared the OS rates of elderly and younger patients in each group. There were no significant differences between elderly and younger patients in the intermediate-, high-, and very high-risk groups ( $\mathrm{P}=0.236,0.841$, and 0.215 , respectively). In the low-risk group, the OS rate of elderly group was significantly lower than that of younger group $(\mathrm{P}=0.00169)$. Meanwhile, there was no significant difference between elderly and young patients in CFFR, even in the low-risk group $(\mathrm{P}=0.443)$.

Grade $\geq 2$ GI toxicity was observed in 23 (9.7\%) cases in the elder group. Among them, six (2.5\%) cases were grade 3 . No cases of grade 4 GI toxicity were observed. The cumulative incidence rates of GI toxicity (grade $\geq 2$ ) at 3-year follow-up were 10.5 and $9.6 \%$ for elder and younger groups, respectively; no significant difference between the two groups was founded ( $\mathrm{P}=0.4143$ ) (Fig. 4). Grade $\geq 2$ GU toxicity was observed in nine $(3.8 \%)$ cases in the elder group. No cases with grade $\geq 3$ GU toxicity were noted. The cumulative incidence 


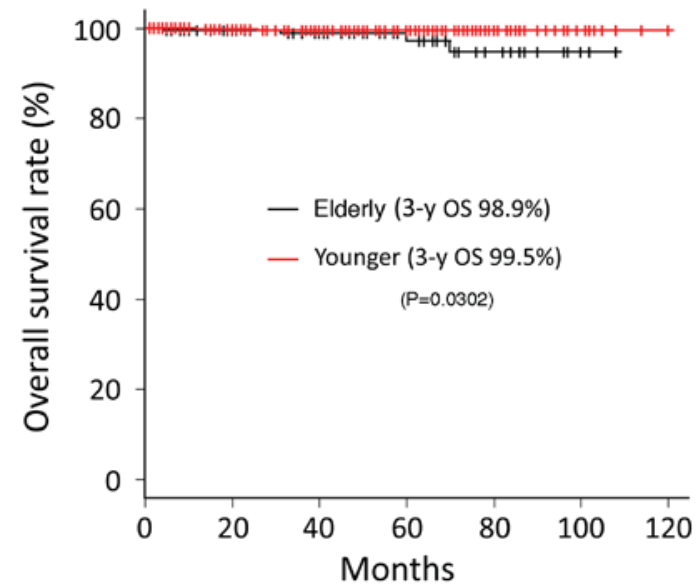

Figure 3. Overall survival rate for the elderly and younger groups. OS, overall survival; y, year.

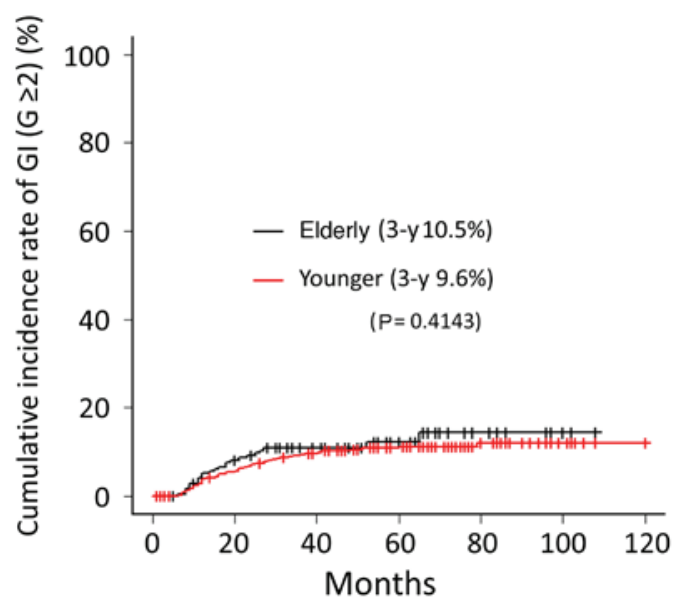

Figure 4. Cumulative incidence rate of Grade $\geq 2$ gastrointestinal adverse events. OS, overall survival; y, year.

rates of GU toxicity (grade $\geq 2$ ) at 3 -year follow-up were 1.3 and $0.6 \%$ in the elder and younger groups, respectively; no significant difference was observed between the two groups $(\mathrm{P}=0.0769)$ (Fig. 5). There were no significant differences in the incidence rates of GI or GU $\geq$ grade 2 overall, or in elderly patients only, due to differences in prescription dose or risk classification.

\section{Discussion}

IMRT has been widely used for EBRT of localized prostate cancer. The outcome of IMRT is comparable to other treatment modalities, such as prostatectomy or brachytherapy (17). Although each modality has advantages and disadvantages, the incidence rate of adverse events in every modality is within acceptable range (18). However, IMRT is the least invasive choice among curable treatment options. To treat cancer in the elderly, the treatment is chosen with consideration of age, life expectancy, general condition, and comorbidity. Elderly patients with prostate cancer were more likely to receive ADT alone (11); however, a study in elderly patients reported that conservative management with ADT alone does not improve the OS rate (12). The life expectancy of the elderly is increasing,

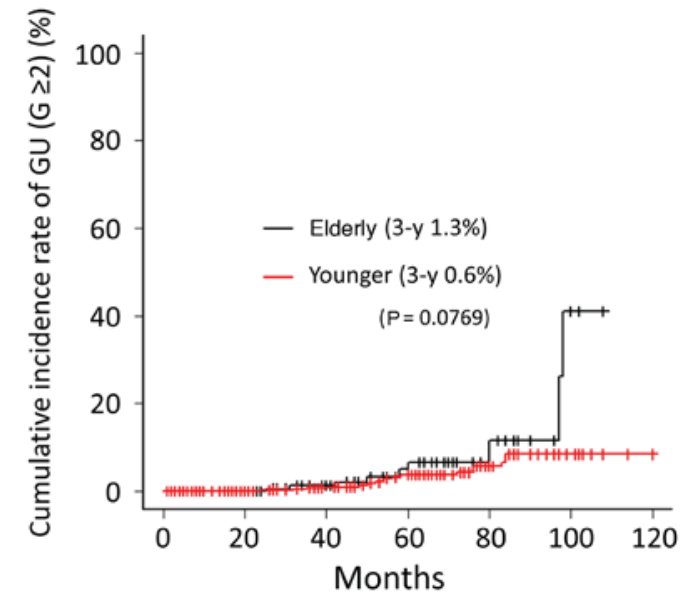

Figure 5. Cumulative incidence rate of Grade $\geq 2$ genitourinary adverse events. OS, overall survival; y, year.

and a curable approach should be considered for patients in good general condition independent of age. IMRT would be the most promising modality among curable treatment choices due to its lower invasiveness. During treatment of elderly cancer patients, a higher frequency and degree of adverse events may be observed (19). It is worthwhile to note that in our study, the IMRT for prostate cancer in the elderly was comparable to that in younger patients. Although no significant difference between elderly and the young patient groups was founded in regard to GU rates, it is necessary to attend to the fact that the incidence rate of GU was higher in the elder group (1.3\% compared to $0.6 \%$ in elder and younger groups, respectively).

In our study, the elder group had a higher overall risk than the younger group. This result is in agreement with the report of Shao et al showing increased proportion of higher risk prostate cancer with increased age at the time of diagnosis (20). Despite the large proportion of high-risk patients, the BFFR and CFFR of the elder group did not significantly differ from those of the younger group. This result may be partly due to the high ADT usage in the elder group; however, the reason for the high usage of ADT was the high proportion of intermediate- and high-risk patients in the elder group. Even regarding adverse events, no significant difference in the incidence rates of both GI and GU could be found between elder and younger groups. The incidence rates of GU tended to increase after five years in both groups; this tendency was particularly strong in the elder group. Therefore, we think that long-term follow-up is necessary even for elderly patients treated with IMRT.

Our study had one major limitation. Since we retrospectively investigated the paper-based medical chart, we could not accurately evaluate any acute adverse events including skin toxicity. The presence or absence of complications was of course confirmed prior to treatment, but we could not accurately record this from the paper-based medical charts.

In conclusion, IMRT has an equivalent treatment effect for prostate cancer in patients aged 75 years or older as in patients younger than 75 years, and the incidence rates for adverse events are also comparable between the two groups. In elderly prostate cancer patients who have good general condition and long life expectancy, IMRT should be considered as a treatment option. 


\section{Acknowledgements}

Not applicable.

\section{Funding}

No funding was received.

\section{Availability of data and materials}

The datasets used and/or analyzed during the current study are available from the corresponding author on reasonable request.

\section{Authors' contributions}

HT, SI, SG, SO and MM conceived and designed the experiments. HT, YN, MI, TY, KE, SK, SI, MH and MM analyzed the data. HT, YN, MI, TY, KE, SK and SG wrote the first draft of the manuscript. HT, YN, MI, TY, KE, SK, SI, MH, SO, SG and MM contributed to the writing of the manuscript, and discussed the results and conclusions. HT, YN, MI, TY, KE, SK, SI, MH, SO, SG and MM jointly developed the structure and arguments for the paper, critically revised the manuscript for important intellectual content, and reviewed and approved the final manuscript.

\section{Ethics approval and consent to participate}

The present study was approved by the Ethics Committee of Kizawa Memorial Hospital. Written informed consents were obtained from the patients.

\section{Patients consent for publication}

Not applicable.

\section{Competing interests}

The authors declare that they have no competing interests.

\section{References}

1. GLOBOCAN 2012: Estimated cancer incidence, mortality and prevalence worldwide in 2012. Leyon: World Health Organization. http://globocan.iarc.fr/Pages/fact_sheets_cancer. aspx. Accessed October 31, 2018.

2. Projected cancer statistics, 2017. Tokyo: Center for Cancer Control and Information Services, National Cancer Center. https://ganjoho.jp/en/public/statistics/short_pred.html. Accessed October 31, 2018.

3. Cancer statistics in Japan 2016. Tokyo: Center for Cancer Control and Information Services, National Cancer Center. https:// ganjoho.jp/en/professional/statistics/brochure/2016_en.html. Accessed October 31, 2018.

4. NCCN Guidelines for treatment of cancer by site. Prostate Cancer. Fort Washington: National Comprehensive Cancer Network. https://www.nccn.org/professionals/physician_gls/pdf/ prostate.pdf. Accessed October 31, 2018.

5. Someya M, Hori M, Tateoka K, Nakata K, Takagi M, Saito M, Hirokawa N, Hareyama M and Sakata K: Results and DVH analysis of late rectal bleeding in patients treated with 3D-CRT or IMRT for localized prostate cancer. J Radiat Res (Tokyo) 56: $122-127,2015$
6. Cahlon O, Zelefsky MJ, Shippy A, Chan H, Fuks Z, Yamada Y, Hunt M, Greenstein S and Amols H: Ultra-high dose (86.4 Gy) IMRT for localized prostate cancer: Toxicity and biochemical outcomes. Int J Radiat Oncol Biol Phys 71: 330-337, 2008.

7. Yamazaki H, Nakamura S, Nishimura T, Yoshida K, Yoshioka Y, Koizumi M and Ogawa K: Transitioning from conventional radiotherapy to intensity-modulated radiotherapy for localized prostate cancer: Changing focus from rectal bleeding to detailed quality of life analysis. J Radiat Res (Tokyo) 55: 1033-1047, 2014.

8. Dolezel M, Odrazka K, Zouhar M, Vaculikova M, Sefrova J, Jansa J, Paluska P, Kohlova T, Vanasek J and Kovarik J: Comparing morbidity and cancer control after $3 \mathrm{D}$-conformal (70/74 Gy) and intensity modulated radiotherapy (78/82 Gy) for prostate cancer. Strahlenther Onkol 191: 338-346, 2015.

9. Tanaka H, Yamaguchi T, Hachiya K, Kamei S, Ishihara S, Hayashi M, Ogawa S, Nishibori H, Goshima S and Matsuo M: Treatment outcomes and late toxicities of intensity-modulated radiation therapy for 1091 Japanese patients with localized prostate cancer. Rep Pract Oncol Radiother 23: 28-33, 2018.

10. Alicikus ZA, Yamada Y, Zhang Z, Pei X, Hunt M, Kollmeier M, Cox B and Zelefsky MJ: Ten-year outcomes of high-dose, intensity-modulated radiotherapy for localized prostate cancer. Cancer 117: 1429-1437, 2011.

11. Bechis SK, Carroll PR and Cooperberg MR: Impact of age at diagnosis on prostate cancer treatment and survival. J Clin Oncol 29: 235-241, 2011.

12. Lu-Yao GL, Albertsen PC, Moore DF, Shih W, Lin Y, DiPaola RS and Yao SL: Survival following primary androgen deprivation therapy among men with localized prostate cancer. JAMA 300: 173-181, 2008.

13. Japan ALTF: Tokyo. Ministry of Health, Labour and Welfare, Japan, 2016. Available from http://www.mhlw.go.jp/english/ database/db-hw/lifetb16/index.html.

14. D'Amico AV, Whittington R, Malkowicz SB, Cote K, Loffredo M, Schultz D, Chen MH, Tomaszewski JE, Renshaw AA, Wein A, et al: Biochemical outcome after radical prostatectomy or external beam radiation therapy for patients with clinically localized prostate carcinoma in the prostate specific antigen era. Cancer 95: 281-286, 2002.

15. Roach MIII, Hanks G, Thames HJr, Schellhammer P, Shipley WU, Sokol GH and Sandler H: Defining biochemical failure following radiotherapy with or without hormonal therapy in men with clinically localized prostate cancer: Recommendations of the RTOG-ASTRO Phoenix Consensus Conference. Int J Radiat Oncol Biol Phys 65: 965-974, 2006.

16. Common Terminology Criteria for Adverse Events (CTCAE) Version 4.0. Bethesda: National Institutes of Healths, Department of Health and Human Services. https://ww w.eortc.be/services/ doc/ctc/CTCAE_4.03_2010-06-14_QuickReference_5x7.pdf. Accessed October 31, 2018.

17. Wilcox SW, Aherne NJ, McLachlan CS, McKay MJ, Last AJ and Shakespeare TP: Is modern external beam radiotherapy with androgen deprivation therapy still a viable alternative for prostate cancer in an era of robotic surgery and brachytherapy: A comparison of Australian series. J Med Imaging Radiat Oncol 59: 125-133, 2015

18. Zelefsky MJ,Poon BY,Eastham J, Vickers A,Pei X and Scardino PT: Longitudinal assessment of quality of life after surgery, conformal brachytherapy, and intensity-modulated radiation therapy for prostate cancer. Radiother Oncol 118: 85-91, 2016.

19. Hayashi S, Tanaka H, Kajiura Y, Ohno Y and Hoshi H: Stereotactic body radiotherapy for very elderly patients (age, greater than or equal to 85 years) with stage I non-small cell lung cancer. Radiat Oncol 9: 138, 2014.

20. Shao YH, Demissie K, Shih W, Mehta AR, Stein MN, Roberts CB, Dipaola RS and Lu-Yao GL: Contemporary risk profile of prostate cancer in the United States. J Natl Cancer Inst 101: 1280-1283, 2009. 